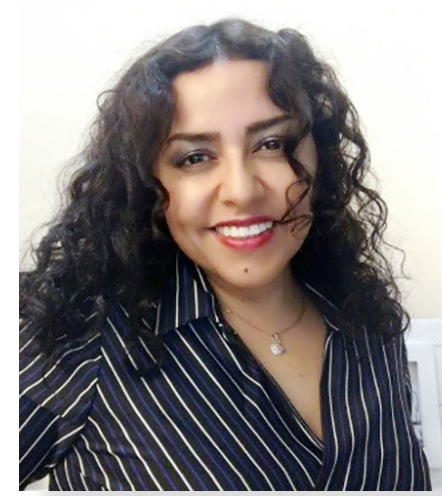

Katia A. Manrique Maldonado

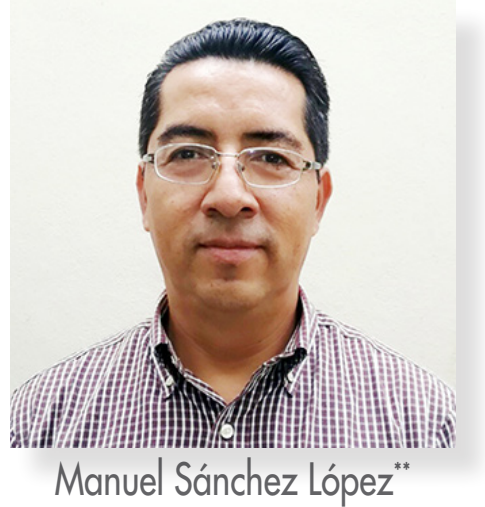

\title{
Satisfacción estudiantil universitaria: un referente para elevar los indicadores de los cursos en línea impulsados por la Coordinación General de Educación Virtual de la UAGro
}

\author{
University student satisfaction: a reference to raise the \\ indicators of the online courses offered by the general \\ coordination of Virtual Education of the UAGro
}

Recibido: 16-09-18

Aprobado: 13-12-18

\section{Resumen}

Las nuevas tecnologías avanzan de manera vertiginosa permeando todas las áreas y ámbitos de la vida, siendo el campo educativo uno de los principales. Debido a esto, la educación del siglo XXI ha transformado en muchos casos el proceso de enseñanza-aprendizaje, sus técnicas, estrategias y herramientas, las cuales se han visto modificadas por el impacto digital. Ante este escenario de evolución constante que genera avances significativos en el entorno educativo se desarrolla el presente artículo, cuyo objetivo es evaluar en distintas variables el nivel de satisfacción de los estudiantes de la Facultad de Ingeniería de la Universidad Autónoma de Guerrero (UAGro) México, con respecto a los cursos impartidos en la modalidad a distancia por la misma universidad. La investigación es de tipo mixta puesto que se utilizaron los beneficios que pueden aportar los enfoques cuantitativos y cualitativos. También es participativa, ya que el estudio cuenta con las voces directas de algunos de los encuestados. Dentro de los resultados se identificó que el proceso de comunicación es una variable importante para la satisfacción del estudiante, ya que los facilitadores ofrecen poca retroalimentación y no son percibidos como motivadores, lo que impide un clima de confianza que contribuya a disminuir la deserción. Al respecto, se detectó que son los monitores académicos los que cumplen una función determinante porque fungen como asesores permanentes que impulsan y estimulan al alumno en el proceso educativo virtual. Los resultados de este estudio podrían contribuir al mejoramiento de los procesos educativos en línea de la UAGro y ser un referente para otras instituciones.

\footnotetext{
*Katia Aleyda Manrique Maldonado: Profesora investigadora de Tiempo Completo de la Universidad Autónoma de Guerrero, México. Dra. en Comunicación Social por la Universidad de la Habana, Cuba; Master en Comunicación Social mención Comunicación Organizacional por la misma universidad. Licenciada en Ciencias de la Comunicación, por la Universidad Autónoma de Guerrero. Para contactar a la autora: katia.manriquem@gmail.com

**Manuel Sánchez López: Docente de Tiempo Completo de la Universidad Autónoma de Guerrero, México. Licenciado en Ciencias de la Comunicación por la Universidad Autónoma de Guerrero. En proceso de titulación de la maestría en enseñanza del Idioma Inglés y Lingüística aplicada en el Centro de Idiomas Extranjeros Ignacio Manuel Altamirano, y en la maestría en Tecnologías Educativas por el Instituto Latinoamericano de la Comunicación Educativa (ILCE). Para contactar al autor: Lopezm601@hotmail.com
} 


\section{Abstract}

New technologies advance in a vertiginous manner permeating all areas and spheres of life, with the educational field being one of the most important. As a result of this, the education of the 21 st century has in many cases transformed the teaching-learning process, its techniques, strategies and tools, which have been modified by the digital impact. This article is developed facing a scenario of continuous evolution that generates significant advances in the educational environment. Its objective is to provide an indicator that shows the level of satisfaction of the students of the Faculty of Engineering of the Autonomous University of Guerrero (UAGro) Mexico, regarding to the courses taught in the distance mode by the same university. The research is a mixed method type since the benefits that quantitative and qualitative approaches can provide were used. Among the results, it was identified that the communication process is an important variable within the student's satisfaction, since the facilitators offer little feedback and they are not perceived as motivators, which prevents having a climate of confidence that contributes to reduce desertion. In this regard, it was detected that the academic monitors are those who play a determining role because they serve as permanent advisors that encourage and stimulate the student in the virtual educational process. The results of this study could contribute to the improvement of the online educational processes of the UAGro and be a reference for other institutions.

\section{Palabras clave}

E- learning; aprendizaje en línea; plataformas virtuales; satisfacción estudiantil

\section{Keywords}

E-learning; online learning; virtual platforms; student satisfaction

\section{Introducción}

La razón de ser de toda universidad es la formación integral de los estudiantes para tener egresados que respondan a las necesidades del entorno, por tanto debe ser una prioridad cuidar la satisfacción de uno de sus grupos de interés más importantes. El conocer su percepción sobre determinados factores proporciona indicadores sobre qué es lo que se debe mejorar o cambiar en ciertos aspectos. De acuerdo con Gento y Vivas (2003), la satisfacción es el resultado de un proceso valorativo. Cuando una persona expresa estar satisfecha o no con respecto a algo, está emitiendo un juicio de valor a partir de un proceso de evaluación. Para los autores, lo anterior transcurre en un proceso de construcción de significados y sentidos personales.

Describir la satisfacción se relaciona con medir la calidad que se brinda al ofrecer un servicio cualquiera que este sea, por tanto, hablar de la satisfacción en los alumnos es hablar de su apreciación sobre el servicio que les brinda la universidad, desde el proceso de enseñanza aprendizaje, la infraestructura, el clima estudiantil, y todo aquello que forma parte de la actividad cotidiana del servicio educativo.

Mejías y Martínez (2009) consideran que “... medir la satisfacción de los estudiantes de una manera consistente, permanente y adecuada, orientaría a la toma de decisiones correctas y así se podrían aprovechar las oportunidades de mejora" (p.32). El proceso de evaluación debe ser constante, lo que permitiría elevar la calidad del servicio educativo al detectar insatisfacciones e inconformidades. Para Salinas, Morales y Martínez (2008), los datos aportados por los estudiantes permiten inferir características importantes del funcionamiento de la institución de la que ellos mismos forman parte y determinan la medida en la que se alcanzan los fines preestablecidos.

Es fundamental tomar en cuenta que los estudiantes están inmersos en un contexto en constante evolución, conforme pasa el tiempo sus expectativas y necesidades cambian, del mismo modo, son diferentes las competencias y conocimientos previos con los que ingresan a un sistema educativo, lo que hace necesario una actualización permanente de planes y programas de estudio acorde a las nuevas expectativas del estudiante y del contexto donde se desenvuelve.

Al respecto, Rodríguez y Pérez (2018) relacionan acertadamente los enfoques teóricos sobre la satisfacción laboral y su vinculación con el sector educativo, tomando entre sus referentes a Maslow con su jerarquía de las necesidades; a Deming con la gestión de calidad; Alderfer con la teoría ERC de la existencia, relación y crecimiento, teoría que toma como base los aportes de Maslow, y la teoría de los dos factores de Herzberg, la cual identifica los elementos que causan insatisfacción y satisfacción laboral. 
De la misma forma, De la Fuente, Marzo y Reyes (2010) toman como referencia a las organizaciones y la satisfacción de los clientes como analogía a la satisfacción dentro del ámbito educativo. No hay que olvidar que las universidades son organizaciones, y que entre sus grupos de interés más importantes se encuentran los estudiantes, docentes y administrativos en lugar de clientes, consumidores, burócratas u obreros.

Al estar la satisfacción ligada a la productividad y al crecimiento de cualquier organización, entre ellas las instituciones educativas con las particularidades que su naturaleza ofrece, es necesario detectar los indicadores que permitirían aportar resultados y tener procesos educativos exitosos. Para Pérez y Pereyra (2015), una medición de la satisfacción en el ámbito universitario puede indicar cómo se está generando el proceso de enseñanza-aprendizaje en la institución, y a lo largo del tiempo podría considerarse como una medida de control de calidad de dicho proceso. En este sentido, son los estudiantes quienes pueden aportar información variada sobre diversos aspectos del servicio educativo que reciben. Conocer su percepción sobre todo lo que concierne a su formación y trabajar con esos indicadores llevará a tener egresados satisfechos y programas más exitosos.

Las universidades tienen como misión formar individuos capaces de desenvolverse de manera exitosa en el campo de trabajo, en un contexto donde los egresados deben de estar acorde a las exigencias del nuevo milenio. Ante esto, las instituciones educativas se ven en la necesidad de integrar los avances en materia tecnológica en sus planes y programas de estudio, asimismo, modificar y actualizar sus contenidos de manera constante para estar a la vanguardia en los procesos educativos. Actualmente, son cada vez más las instituciones que rompen la barrera de la distancia geográfica y ofertan sus planes de manera virtual, a través de plataformas digitales en las cuales la interacción alumno-docente cambia, como cambian la forma de presentar los contenidos, diseñar el proceso de evaluación, fomentar la retroalimentación y las formas y canales de comunicación utilizados.

Por todo lo expuesto, los objetivos del presente artículo son evaluar el nivel de satisfacción de los estudiantes de la Facultad de Ingeniería de la Universidad Autónoma de Guerrero (UAGro) México, con respecto a los cursos impartidos en la modalidad a distancia por la misma universidad; identificar las variables que afectan la satisfacción de los estudiantes sobre los cursos en línea, así como el impacto en la comunicación entre docentes y estudiantes en el proceso de enseñanza aprendizaje en línea.

De los programas educativos que oferta actualmente la UAGro se tomó a la Facultad de Ingeniería como objeto de estudio. Su visión establecida es mantener el liderazgo académico regional, con programas educativos acreditados y procesos administrativos certificados que impacten en el desarrollo productivo sustentable. Actualmente cuenta con cuatro programas educativos: Ingeniero Civil, Ingeniero Constructor, Ingeniero en Computación, Ingeniero Topógrafo y Geomático y la Maestría para la Innovación y Desarrollo Tecnológico. Se consideró esta facultad como objeto de estudio por ser una de las más grandes de la Universidad y por cumplir con los estándares que se les exige a instituciones de nivel superior en el país.

De la misma forma, se trabajó con la Coordinación General de Educación Virtual de la UAGro (CGEV), responsable de los cursos en línea. Esta coordinación entró en funciones en el mes de septiembre 2013, a través de un acuerdo con el Sistema Nacional de Educación a Distancia (SINED) que consistió en la instalación de un nodo periférico en el Estado de Guerrero y un servidor con plataforma MOODLE. Actualmente oferta el bachillerato en línea, diplomados, cursos y talleres, así como, dos licenciaturas.

Los resultados proporcionarán un indicador sobre la percepción y satisfacción de los estudiantes sobre el servicio educativo, lo que permitirá reforzar el trabajo de esta dependencia, la toma de decisiones, elevar la satisfacción estudiantil en el desarrollo de los cursos en línea y disminuir el porcentaje de deserción al detectar las necesidades y barreras a las que se enfrentan los estudiantes.

Es importante recalcar que es el primer estudio sobre el tema en la Universidad y la CGEV hasta el momento no ha implementado instrumentos que permitan identificar necesidades, problemáticas ni el nivel de satisfacción de los estudiantes que toman un curso en línea, a pesar de que los objetivos a futuro son llevar más unidades de aprendizajes y programas educativos presenciales al ámbito digital.

El artículo inicia abordando conceptualizaciones sobre investigaciones que han tratado los temas de satisfacción universitaria, las plataformas virtuales en escenarios educativos, el análisis del proceso de comunicación ante estudiantes denominados "nativos digitales" y se retoma el constructivismo social como una teoría educativa presente en el proceso de enseñanza actual. En segundo lugar, se describe la metodología mixta utilizada como eje rector de la investigación, asimismo, se presentan los resultados divididos en las siguientes categorías de análisis: Tecnología, Comunicación en el proceso de enseñanza aprendizaje, Desempeño docente, Desempeño del estudiante, Evaluación, y Aprendizajes esperados. Finalizamos con las conclusiones y recomendaciones

«Satisfacción estudiantil universitaria: un referente para elevar los indicadores de los cursos en línea impulsados por la Coordinación General de Educación Virtual de la UAGro», Katia Aleyda Manrique Maldonado y Manuel Sánchez López. Cuaderno de Pedagogía Universitaria Vol. 16 / no. 31 / enero-junio 2019 / República Dominicana / PUCMM / ISSN 1814-4152 (en línea) / pp. 17-30 
para lograr un proceso en línea eficaz a partir de fortalecer el rol del docente facilitador y su oportuna retroalimentación.

\section{Nuevos escenarios educativos en el siglo XXI}

Las teorías del aprendizaje explican y describen, entre otras cosas, cómo se desarrolla el aprendizaje en el ser humano y cómo se apropia este del conocimiento, del mismo modo estudian su comportamiento en diversos procesos y entornos. Para Hernández (2007), los expertos en diseño educativo en línea deben contar con especialistas en pedagogía conocedores de las bases del constructivismo, ya que, de acuerdo con su postura, en esta era del conocimiento compartido el trabajo cooperativo en la integración de grupos inter- e intradisciplinarios cobra mayor relevancia.

Los nuevos escenarios implican la incorporación de diferentes recursos tecnológicos, uno de ellos son las plataformas virtuales que se han popularizado para incorporarlos a programas educativos de todos los niveles, con el enfoque de E-Learning o B-Learning. Para Sebastián Díaz, una plataforma educativa virtual "es un entorno informático en el que nos encontramos con muchas herramientas agrupadas y optimizadas para fines docentes. Su función es permitir la creación y gestión de cursos completos para internet sin que sean necesarios conocimientos profundos de programación" (2009, p.2).

Una de las plataformas ampliamente utilizada dentro del sector educativo es Moodle, creada por Martin Dougiamas a partir del constructivismo social. Está diseñada para el sector educativo de forma gratuita; su primera versión data del año 2002. Esta plataforma crea un ambiente de aprendizaje virtual y colaborativo donde los docentes y alumnos interactúan de una manera dinámica y significativa (Moodle, 2018). Actualmente, una gran cantidad de programas de todos los niveles cuelgan sus contenidos en esta plataforma.

El sitio de Moodle (2018) expone dentro de su filosofía que esta plataforma está fundamentada en "cuatro conceptos principales subyacentes" el constructivismo, el construccionismo, el constructivismo social y conectados y separados, este último considerado como una idea que explora la importancia de la reflexión del individuo y las opiniones y puntos de vista de los demás. Con ello se logra una interacción y aprendizaje más significativo porque permite que cada participante en algún momento pueda ser maestro en lugar de alumno, el trabajo del profesor puede cambiar de ser la fuente de la información de la que dependen a un tutor, con una relación con los estudiantes de manera más horizontal y personal.
El constructivismo considera que el estudiante se apropia del conocimiento mediante una construcción que se genera a partir de las experiencias en su contexto y de su desarrollo cognitivo, de esta forma el individuo podrá estructurar de manera permanente nuevos aprendizajes. Este enfoque está sustentado en corrientes psicológicas y educativas por teóricos como Lev Vygotski, Jean Piaget, David Ausubel, y Jerome Bruner y viene a contraponerse al trabajo de la educación tradicional y sus características presentes por muchos años en las aulas de todos los niveles.

El teórico norteamericano Ausubel desarrolló la teoría del aprendizaje significativo. De acuerdo a esta propuesta, para aprender son importantes los conocimientos previos del estudiante para darle significado a los nuevos conocimientos de los que se van apropiando. Para conseguirlo, es determinante que el estudiante llegue a complementar la relación de los aprendizajes previos con los nuevos. Ausubel, citado por Tünnermann (2011, p. 24), definió tres condiciones básicas para que se produzca el aprendizaje significativo:

- que los materiales de enseñanza estén estructurados lógicamente con una jerarquía conceptual, situándose en la parte superior los más generales, inclusivos y poco diferenciados;

- que se organice la enseñanza respetando la estructura psicológica del alumno, es decir, sus conocimientos previos y sus estilos de aprendizaje;

- que los alumnos estén motivados para aprender.

De igual forma, para el autor norteamericano los aspectos motivacionales influyen de manera determinante para producir un aprendizaje significativo. Barraza (2002), por su parte, considera que se ha logrado un aprendizaje cuando el alumno le puede atribuir al contenido un significado, y esto solamente sucede si el alumno consigue establecer relaciones sustantivas y no arbitrarias entre el nuevo material de aprendizaje y sus conocimientos previos.

Es necesario observar que en un proceso educativo en línea el estudiante y el docente se enfrentan a una modalidad de enseñanza-aprendizaje donde el contexto educativo tradicional cambia radicalmente, en muchos casos el salón de clases se convierte en un aula virtual y existe la posibilidad de que el maestro y el estudiante no lleguen a conocerse cara a cara en todo el proceso.

Hernández (2008) considera que el docente de cursos virtuales, como asesor constructivista en línea, ha de desarrollar múltiples actividades, que entre otras cosas, implican: reflexionar acerca de su grado de compromiso no solo con sus estudiantes, sino consigo mismo; 
saber programar acciones interactivas; reconocer que no todos aprenden de la misma forma ni al mismo ritmo; propiciar la participación social y los hábitos de estudio entre los alumnos y salvar progresivamente las dificultades con el uso de la computadora. El trabajo del docente se ve modificado por un entorno distinto, herramientas diferentes y estudiantes del nuevo milenio, elementos que impactan en su ejercicio diario debido a que un aprendizaje en un entorno virtual dista mucho de uno que se desarrolla en el ambiente tradicional.

Dentro de un aprendizaje que se desarrolla en ámbitos virtuales o digitales, el lenguaje y sus significados se ven modificados, la construcción del conocimiento en el siglo XXI cambia ante el uso de diferentes tecnologías de la información y la comunicación que hoy en día están al alcance de los alumnos. El video, los libros, el material multimedia, los exámenes, las ilustraciones y demás recursos educativos tienen que abordarse desde diferentes perspectivas y adaptarse a un lenguaje y significados que se han modificado con el paso del tiempo gracias al uso y contacto con las nuevas tecnologías.

Para Díaz y Hernández (2010), un profesor constructivista es un mediador entre el conocimiento y el aprendizaje de sus alumnos; es un profesional reflexivo; promueve aprendizajes significativos y la colaboración; establece como metas la autonomía y la autodirección; analiza críticamente sus propias ideas y presta una ayuda pedagógica ajustada a la diversidad de necesidades educativas por las que atraviesan sus alumnos.

\section{La comunicación dentro del proceso de enseñanza aprendizaje del siglo XXI}

La comunicación se ha ido adaptando a los diferentes cambios tecnológicos, que día a día han aparecido en la vida cotidiana del hombre, alterando la forma de vivir, convivir y relacionarse con los otros. Linares y Santovenia (2012, p.15) dicen que "es importante identificar a la comunicación como un conjunto de palabras, ideas, mensajes o discursos, que desde diferentes formas expresivas aportan información y conocimiento". Los autores visualizan a la comunicación como un hecho sociocultural producto de la relación entre individuos.

Los elementos básicos del proceso de comunicación: emisor, mensaje, receptor y retroalimentación cumplen una función determinante y circular en la reciprocidad de la información. La educación no puede aislarse de la revolución en la comunicación y la tecnología que está permeando todos los ámbitos y exige, sin lugar a dudas, modificar los procesos de comunicación en el contexto educativo, así como las metodologías pedagógicas tradicionales para adaptarlas a las necesidades del nuevo milenio. (Manrique, 2015)
García Aretio (1999), por su parte, enfatiza que la interacción en la educación a distancia puede adoptar diversas modalidades en función de la intermediación, del tiempo y del canal. En función de la intermediación puede ser presencial (cara a cara) o no presencial (mediante algún tipo de canal). En función del tiempo: síncrona (cuando se da en tiempo real o simultánea e inmediata a la producción del mensaje); asíncrona (la retroalimentación no se realiza de forma simultánea, sino diferida por minutos, horas o incluso días). En función del canal, puede ser real (es asíncrona o síncrona, pero objetiva, mediante cualquier canal) o virtual (cuando hay un contacto solo con el material destinado para el aprendizaje, como el ejemplo el material multimedia o los servicios de Internet).

Del manejo que los docentes hagan de las diferentes herramientas, será el nivel de efectividad de la comunicación con sus estudiantes en el entorno virtual, de ahí la importancia de conocerlas para saber el impacto de cada una de ellas y cuándo utilizarlas.

Alonso y Blázquez (2012) recomiendan que es necesario utilizar aquellos medios que se consideran convenientes para cada tipo de mensaje y recomiendan, entre otras cosas, no basarse en la novedad de la herramienta, sino en su pertinencia; elegir solo aquellos medios que sean funcionales; preferir medios sincrónicos o asincrónicos de acuerdo a las características del objetivo pedagógico que se pretenda conseguir; prever con anticipación la planeación de cada herramienta para gestionarla adecuadamente y así poder controlar los resultados del aprendizaje.

El papel del docente tutor tiene una particular importancia al interior de un proceso de aprendizaje virtual, debido a que este es responsable de dinamizar los espacios de comunicación desarrollando habilidades que permitan motivar a los alumnos y empatizar con sus necesidades; ofrecer retroalimentación para captar la atención de los desmotivados y desarrollar una habilidad especial para saber interpretar y comunicarse a través de elementos característicos de la red como son los emoticones, podcasts y webcasts. (Alonso y Blázquez, 2012).

Ante estos cambios tecnológicos constantes que impactan los procesos educativos en todos sus niveles, surgen cada día nuevos términos y conceptos para definir a las nuevas generaciones dotadas de competencias y conocimientos tecnológicos. Prensky (2001) acuñó los términos de Nativos e Inmigrantes Digitales de acuerdo con las características presentes en los estudiantes del nuevo milenio. El investigador considera como nativos digitales a las generaciones que nacieron aproximadamente desde los años noventa hasta nuestros días y para quienes los diversos avances tecnológicos como son las computadoras, los dispositivos móviles y el Internet son 
de uso frecuente. Para estos estudiantes la tecnología es parte de su vida cotidiana y la incluyen a sus diferentes procesos educativos. Las personas nacidas antes de los años ochenta son denominadas por Prensky (2001) como inmigrantes digitales, y son aquellos que se han ido incorporando, en algunos casos con más dificultad, a esta vorágine tecnológica que ha envuelto el mundo contemporáneo.

Pisani y Piscitelli, citados por Acuña (2012), refieren características diferenciadoras en los nativos digitales: la creación de un nuevo lenguaje en el cual se evidencia una incorrecta ortografía, gramática y sintaxis; la multisensorialidad: utilizan casi todos los sentidos a la vez, la vista, el oído y el tacto, y el pensamiento nolineal: esa capacidad de estar en contacto con otros jóvenes a través de universos virtuales; el uso de internet utilizando hipertextos y la facilidad de "navegar" de una manera diversa y activa. Este universo creciente de aparatos tecnológicos, el desarrollo de internet y sus múltiples funciones y oportunidades han generado un nuevo lenguaje digital del cual los nativos digitales se apropian, construyen y modifican.

Los procesos de comunicación entre los jóvenes han incorporado un nuevo tipo de "lenguaje digital" derivado del uso e interacción constante con las tecnologías que los identifica y distingue cada vez más, y con el cual los inmigrantes digitales no están tan familiarizados. Para Piscitelli "La disyunción es clara: o los inmigrantes digitales aprenden a enseñar distinto o los nativos digitales deberán retroceder sus capacidades cognitivas o intelectuales a las que predominaban dos décadas y más atrás." (2009, p.48) En este aspecto, es claro que los docentes tienen que actualizar sus programas y sus competencias a las necesidades del estudiante y dominar los procesos de comunicación adoptando el lenguaje y nuevos códigos manejados por sus alumnos, de lo contrario, será cada vez más difícil que los procesos de enseñanza-aprendizaje tengan el éxito esperado.

Prensky (2001) considera que los estudiantes de este nuevo milenio no son las personas que corresponden al diseño del sistema educativo tradicional. Hay que considerar que las características de los estudiantes de hace 20 años no son las de los estudiantes actuales debido al acelerado avance e impacto de las tecnologías, aunque en un mismo país las condiciones económicas y sociales no son las mismas para todos. Mientras que algunos nacen en un ambiente donde el uso e incorporación de los avances tecnológicos es algo común con acceso a videojuegos, teléfonos celulares, computadoras e internet, en otros contextos de precariedad les resulta difícil, sino imposible, incorporar las últimas novedades al respecto, ya que en ocasiones apenas cuentan con acceso a los servicios básicos.
Elena Gallardo (2012) da sus conclusiones al respecto: "en primer lugar, no hay una definición absoluta de los nativos digitales ya que puede variar entre los individuos, sociedades, regiones y naciones, y a través del tiempo. En segundo lugar, nacer en la era digital no implica una mayor competencia digital" (p.16). La autora sostiene que se ha encontrado que no hay suficiente evidencia para demostrar que los nativos digitales son digitalmente competentes y que estas habilidades sean transferidas al ámbito académico. Por este motivo, es importante visualizar también que no toda una generación cumple con las mismas características y el acceso a la tecnología depende de las características socioeconómicas de los países y sus regiones. A pesar de esto, el docente en línea tiene la responsabilidad de actualizarse de manera permanente ante los recursos tecnológicos y desde el punto de vista metodológico, para que se reduzcan los efectos de esta brecha generacional y se establezca una interacción más dinámica y un feedback efectivo acorde a las necesidades de los estudiantes en entornos virtuales.

\section{Metodología}

Esta investigación es de tipo mixta ya que se tomaron los beneficios que pueden aportar los enfoques cuantitativos y cualitativos. Es también participativa, pues siempre que lo consideramos oportuno, introducimos la opinión de los participantes. Esto nos permitió obtener un análisis e interpretación de los datos de una manera más profunda, mediante la convergencia y triangulación de los resultados, asimismo, obtener datos más objetivos por la variedad en las técnicas de recolección utilizadas. Al contrastar la información obtenida, se logró ahondar en el tema de una manera más clara que utilizando uno de los dos diseños. Hernández, Fernández y Baptista (2014) exponen que la triangulación se refiere a verificar convergencia, confirmación o correspondencia al diferenciar datos cuantitativos y cualitativos, así como a corroborar o no los resultados y descubrimientos para alcanzar una mayor validez interna y externa de la investigación.

La aplicación de las técnicas fue simultánea y secuencial, utilizando las mismas categorías para poder llegar a una comparación de los resultados. En el desarrollo del trabajo ambos diseños tuvieron la misma importancia para el estudio.

Por lo tanto, las técnicas cuantitativas para la recolección de información, como la encuesta, nos proporcionaron un panorama más amplio sobre la satisfacción de los estudiantes con respecto al curso en línea, así como también las características del diseño cualitativo al profundizar con sus entrevistas semiestructuradas y la observación la eficacia del trabajo de los estudiantes en la plataforma digital. 


\section{Contexto y delimitación de la investigación:}

La investigación se llevó a cabo en la ciudad de Chilpancingo, dentro de la Facultad de Ingeniería de la Universidad Autónoma de Guerrero, México, con estudiantes que tomaron por lo menos una Unidad de Aprendizaje en la modalidad Virtual durante el semestre febrero-julio 2017 y agosto 2017 - enero 2018.

\section{Población y muestra}

De acuerdo a la información proporcionada por la UAGro Virtual para el ciclo escolar que se estudió, hubo 209 alumnos de la Facultad tomando por lo menos uno de los cursos que se ofertan en línea.

El tamaño de la muestra para un margen de error del \pm 5 y un nivel de confianza del $95 \%$ da como resultado 136 cuestionarios utilizando la fórmula proporcionada por el sitio web SurveyMonkey (2017), y The Survey System (2017), igualmente se tomó como referencia la tabla estadística de Arkin y Colton (Ruiz, 2008), de esta forma se optó por aplicar 142 cuestionarios. Los resultados fueron procesados en el programa estadístico informático Statistical Package for the Social Sciences (SPSS) versión 18.

La selección de las personas entrevistadas fue de acuerdo a la pertinencia y vínculo con el tema de investigación. Los nombres utilizados en el desarrollo del artículo fueron suprimidos y en otros casos alterados por cuestiones de confidencialidad.

\section{Resultados}

\section{Tecnología}

Para ofrecer el servicio educativo la Coordinación General de Educación Virtual trabaja con MOODLE. La Plataforma Learning Management System, permite la interacción digital activa entre facilitadores $y$ estudiantes, los cuales pueden a través de ella compartir información, debatir, desarrollar conocimientos y ser evaluados en cada una de sus unidades de aprendizaje. Dentro de todos los planes de estudio de nivel superior de la UAGro, se encuentra la Etapa de Formación Institucional (EFI) que incluye 6 unidades de aprendizaje o tronco común, que se trabajan de manera presencial y a la vez se ofertan de manera virtual. Estas son Inglés I y II, Análisis del mundo contemporáneo, Manejo de las Tecnologías de Información y Comunicación, Habilidades para la Comunicación de las Ideas, y Pensamiento Lógico, Heurístico y Creativo. El 49.3\% de los estudiantes encuestados pertenecientes a la
Facultad de Ingeniería han tomado por lo menos una unidad de aprendizaje en línea. El $24.06 \%$ ha tomado dos unidades de aprendizaje, y el $14.1 \%$ han tomado tres unidades. El $45.8 \%$ de los estudiantes que emitieron su opinión tienen entre 18 y 20 años, el $33.8 \%$ entre 21 y 22 años y el $20.5 \%$ más de 23 años.

\section{Funciones y uso de la plataforma}

El uso de las tecnologías no es siempre fácil para todos los estudiantes, al preguntarles sobre el manejo de la plataforma, el $46 \%$ de ellos contestó que fue sencillo trabajar con ella; el 19\% manifestó que fue muy sencillo; al $11.3 \%$ le pareció complicado y el $22.5 \%$ dijo que fue medianamente complicado.

Omar García Rodríguez, estudiante del grupo 801 del programa educativo Ingeniero Constructor, manifestó al respecto lo siguiente: "es muy complicado meterse a la plataforma, luego tienes que abrir tu correo y aparte es complicado enviar las tareas porque a veces te confundes... la verdad no me gustó nada". Manuel J. Hernández, estudiante del séptimo semestre del programa educativo Ingeniero en Computación, opinó que el manejo de la plataforma fue "Un poco confuso", al igual que Roberto Rodríguez M. del programa educativo de Ingeniería Civil, el cual consideró a la plataforma como "Confusa". Sin embargo, José Marino Cabrera, del programa educativo Ingeniero Constructor del tercer semestre, comentó que "fue muy sencillo ya que al principio de entrar a una Unidad de Aprendizaje siempre nos ayudaron en ese proceso".

Al preguntar sobre el diseño de la plataforma: colores, fotos, texto, títulos, videos, al $46.5 \%$, le parece bueno y al $26.1 \%$ le pareció excelente. Ver figura 1

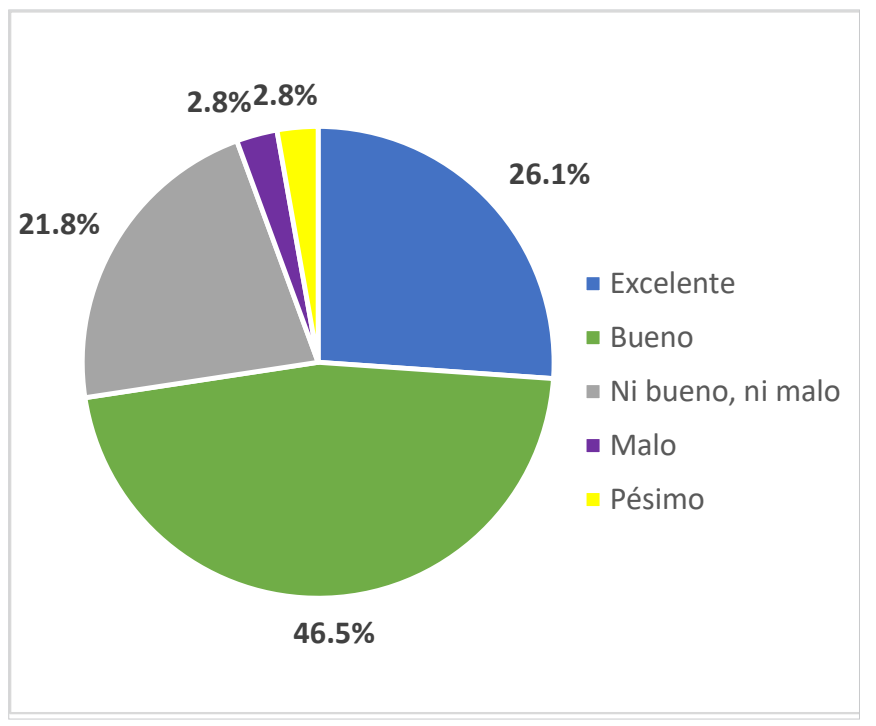

Figura 1: El diseño de la plataforma colores, fotos, texto, títulos, imágenes y videos te parece... 
Sobre el punto de vista de la calidad de los materiales encontrados en la plataforma, como lecturas, documentos, videos, etc., el $40.1 \%$ manifestó que frecuentemente había calidad en ellos, mientras que un $26.1 \%$ dijo que muy frecuentemente. Sin embargo, existe, aunque menor, un porcentaje de estudiantes, $17.6 \%$, que consideran que solo ocasionalmente; $1.4 \%$ opina que nunca y el $4.9 \%$ no contestó. Ver figura 2

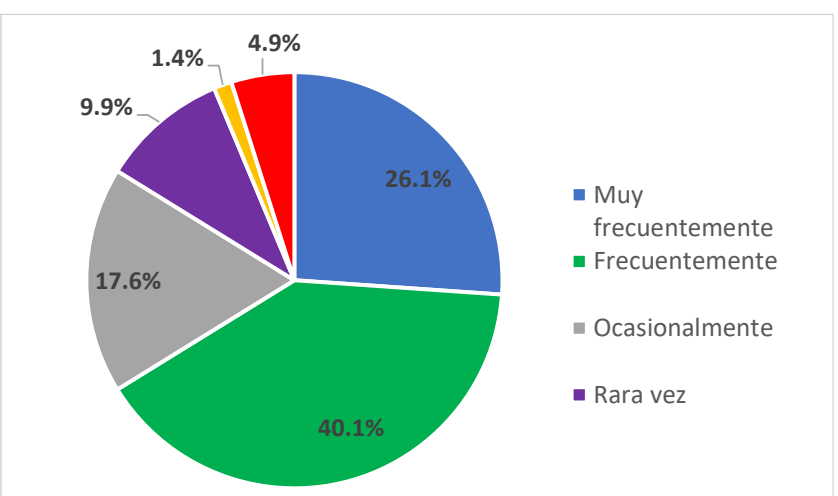

Figura 2: ¿Consideras que hay calidad en los materiales encontrados en la plataforma como vídeos, figuras y documentos?

Los estudiantes encuestados han tomado diferentes unidades de aprendizaje en línea las cuales manejan materiales distintos. Al respecto, de los doce estudiantes entrevistados, 9 manifestaron que los contenidos eran de calidad, mientras que tres consideraron que les faltaba, Jorge Martínez Castro, estudiante del programa educativo Ingeniero en Computación, opinó: "Sí me servían, pero no eran de la mayor calidad, pero sí eran buenos porque nos permitían trabajar".

Al preguntar cómo percibían la distribución e instrucciones de las unidades de aprendizaje en la plataforma, el $50.7 \%$ de estudiantes encuestados le pareció sencillo, mientras que el $22.5 \%$ le pareció medianamente complicado y el $14.8 \%$ manifestó que complicado. Se indagó sobre si les parecía el contenido del curso dentro de la plataforma fácil de ubicar, a lo que el $44.4 \%$ manifestó que frecuente mente, para el $21.8 \%$ muy frecuentemente, y el $20.4 \%$ consideró que ocasionalmente. Ver figura 3

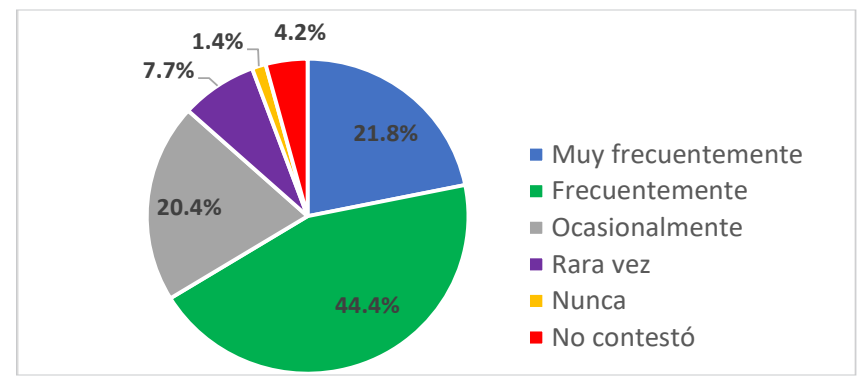

Figura 3: ¿ El contenido del curso dentro de la plataforma es fácil de ubicar?
Es importante considerar que una de las problemáticas que los estudiantes enfrentaron es que algunos se les dificultaba contar con los recursos necesarios para estudiar una unidad de aprendizaje en línea como lo es la computadora y acceso a internet. Esto puede influir en cómo se perciben ciertos aspectos de la plataforma. Ver figura 4

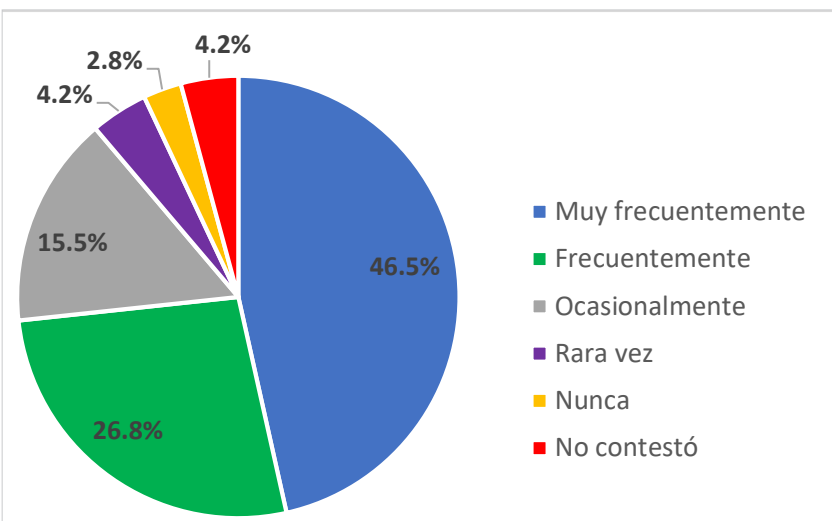

Figura 4: ¿ Contabas con el equipo de cómputo adecuado para trabajar el curso en línea?

La alumna Sofía Sánchez López del programa educativo Ingeniero en Computación, al preguntarle cuál era una de las problemáticas al tomar una unidad de aprendizaje virtual comentó que "la falta de internet, o que a veces se descomponía mi computadora y no podía entregar mis actividades a tiempo" Con respecto al envío de tareas y trabajos mediante la plataforma fue para el $47.9 \%$ sencillo, para el $23.2 \%$ muy sencillo, para el $18.3 \%$ lo consideró medianamente complicado, el $7.7 \%$ complicado, el $2.1 \%$ muy complicado y el $7 \%$ no contestó.

\section{Comunicación dentro del proceso de enseñanza aprendizaje}

La retroalimentación es el objetivo primordial en el proceso de comunicación, dentro del esquema de enseñanza-aprendizaje, el contacto alumno-docente es fundamental para que el proceso de la comunicación sea exitoso, si es frecuente, activo y eficaz contribuye a cumplir con las expectativas del estudiante. En este ítem el $40.1 \%$ considera que lograr el contacto con el facilitador mediante la plataforma fue sencillo; el $13.4 \%$ manifestó que fue muy sencillo; no obstante, un porcentaje considerable contestó lo contrario. El 20.4\% lo calificó como medianamente complicado,18.3\% como complicado y el $6.3 \%$ como muy complicado. Ver figura 5. 


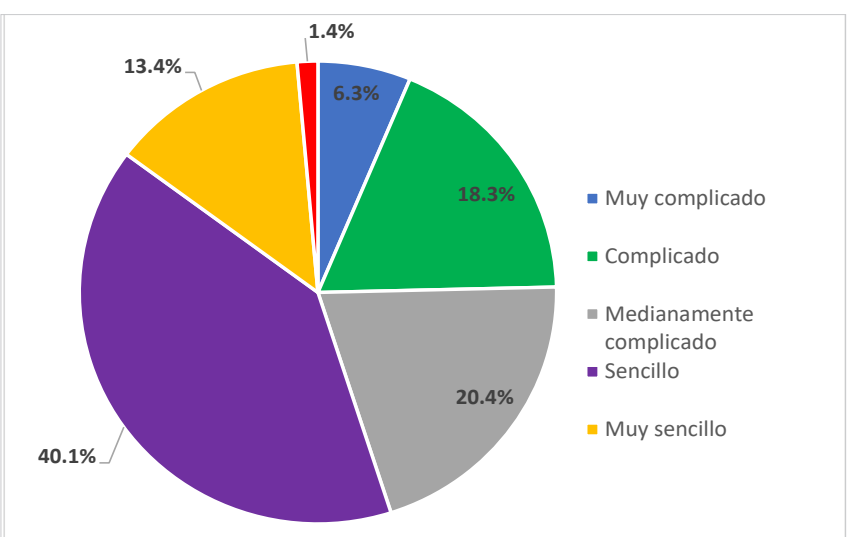

Figura 5: ¿En que grado de complejidad se da el contacto entre docente y estudiante?

Es importante mencionar que son distintos docentes y dependen de la unidad de aprendizaje que el estudiante esté tomando. Respecto a la frecuencia con que se daba este contacto, los porcentajes más representativos no fueron tan positivos ya que el $36.6 \%$ dijo que ocasionalmente; el $31.7 \%$ opinó que rara vez y un $7.0 \%$ que nunca, en porcentajes bajos se encuentra muy frecuentemente $4.2 \%$ y frecuentemente con un $16.2 \%$ Ver figura 6.

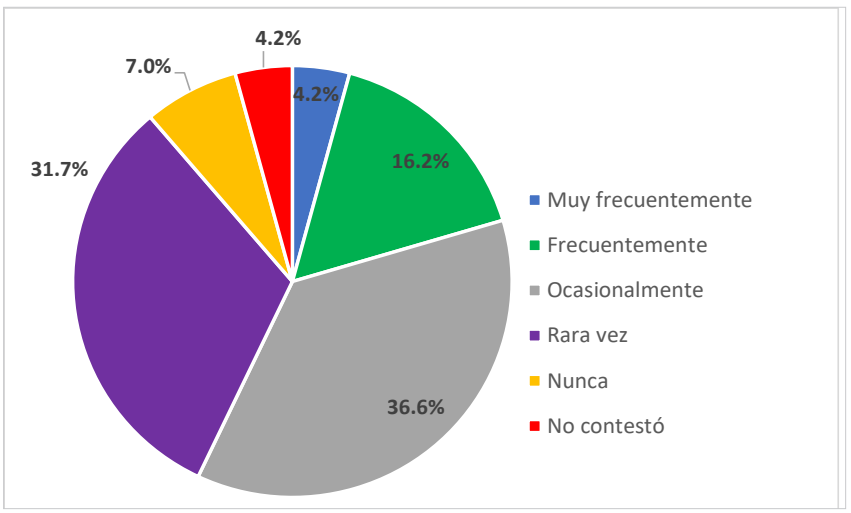

Figura 6: ¿Con que frecuencia se da el contacto con el docente?

Por tanto, se puede detectar en los resultados una debilidad en la comunicación entre facilitadores y estudiantes desde cómo se percibe el contacto entre ellos dentro de la plataforma hasta la frecuencia con que se produce esta.

Al preguntar a los estudiantes entrevistados cómo fue la retroalimentación con el facilitador, estas fueron algunas de sus respuestas: Roberto Rodríguez $\mathrm{M}$. del programa educativo de Ingeniería Civil dijo: "Mala, nunca respondió mis dudas, me decía que leyera las instrucciones las cuales no eran claras." Omar Sánchez Solís, del programa educativo Ingeniero Constructor, opinó al respecto: "Rara vez, casi no interactuamos". Para Martín Soto Molina, del programa educativo
Ingeniero en Computación, su experiencia con el docente la cataloga como buena: "muy bien, explica muy bien, te da las indicaciones debidas, hasta ahorita no ha habido problemas con él en ese aspecto porque si explica"

En la Coordinación General de Educación Virtual existe la figura del monitor académico que puede ser también un canal e intermediario entre el facilitador y el estudiante, esta función la desempeña una persona que debe estar atento del desarrollo del estudiante y funge a la vez como guía para aclarar sus dudas. Gonzalo C. Contreras, monitor académico, comenta que dentro de las funciones del monitor está orientar, guiar, motivar y apoyar a superar obstáculos de orden cognitivo y afectivo para aminorar la soledad y, sobre todo, evitar el desánimo de los estudiantes, que no sientan que están solos". Para José C. Ortega es "dar seguimientos y apoyo a los estudiantes, así como también a los facilitadores en cuestiones técnicas que tienen que ver con la plataforma educativa en donde se imparten las materias". Actualmente están registradas seis personas como monitores académicos dentro del sitio web.

Arnaldo L. Martínez, monitor académico, expuso al respecto: "Por mi parte soy consciente de que existen algunos maestros que no hacen sus respectivas retroalimentaciones porque he observado y he tenido varios cursos con varios maestros diferentes y algunos no les dan retroalimentación o la retroalimentación es muy poca, pero hay otros maestros, que si les dan la retroalimentación correspondiente y les dan sus observaciones dependiendo de su trabajo."

\section{Canales y barreras de comunicación}

La coordinadora general de Educación Virtual, al respecto de los canales de comunicación, comentó en una entrevista que las unidades de aprendizaje están basadas en su modelo académico pedagógico y el diseño instruccional y la manera de comunicarse es a través del correo, los foros o grupos de Facebook para cualquier duda que tenga el estudiante, o lo puede hacer a través de un chat en la plataforma. Las respuestas se les pueden dar antes de que se cumplan las 24 horas.

Los tres monitores académicos entrevistados coinciden que el medio más efectivo para comunicarse con los estudiantes es el WhatsApp pues se pueden contactar de una manera más rápida con ellos y el medio de comunicación menos efectivo es el correo electrónico porque los estudiantes no lo responden. José C. Ortega, monitor académico, comentó al respecto: "aunque el correo electrónico anteriormente era una herramienta muy buena, yo he visto que actualmente 
los jóvenes ya no recurren mucho a él, están más inmiscuidos en las redes sociales". En el mismo sentido, mencionó que la comunicación con los estudiantes que están fuera del área geográfica es solamente virtual, pero los estudiantes que radican en la misma localidad donde se encuentran las instalaciones pueden acudir de manera presencial por alguna duda. Para el monitor Arnaldo L. Martínez, el medio menos efectivo es la misma plataforma "porque hay días que entran (los estudiantes) y hay días que no. No entran en tres días hasta que yo les envió un mensaje y me vuelven a responder a los tantos días o cuando ya pasó el tiempo de la actividad..."

Con respecto a los foros como medio de comunicación, José C. Ortega afirma que se cuenta con foros de participación o foros de dudas donde el facilitadormonitor los apertura y se estructura una pregunta detonadora, se puede comentar algún tema en particular o puede ser un medio para resolver dudas sobre un proyecto o investigación que estén realizando.

Una de las problemáticas a las que se enfrentan los estudiantes es no estar familiarizados con el uso de las herramientas tecnológicas, por tal motivo se les proporciona un curso de inducción donde se les enseña el manejo de herramientas que utilizarán en la plataforma.

\section{Desempeño docente}

De acuerdo a información proporcionada por la Coordinación de Educación Virtual, el número de docentes oscila entre 25 y 33 conforme a la demanda, porque los grupos virtuales se abren siempre y cuando haya suficientes estudiantes.

A la pregunta de ¿cómo fue impartida la unidad de aprendizaje de manera virtual?, $10.6 \%$ de los estudiantes opinó que excelente; $62.7 \%$ le pareció bueno el curso; $21.8 \%$ dijo ni bueno ni malo; el $2.8 \%$ dijo que malo y un $2.8 \%$ que pésimo.

¿Cómo evaluarías a los docentes? Es una pregunta que nos permitió tener una retroalimentación sobre la percepción más directa del desempeño del facilitador. El $42.3 \%$ los evalúa como buenos maestros; el $21.1 \%$ como excelentes; un $28.9 \%$ de los estudiantes opina que no son ni buenos, ni malos; un $6.3 \%$ opina que son malos y un $1.4 \%$ dice que son pésimos. Ver figura 7

¿El docente despejó sus dudas durante el curso? Esta pregunta nos ayuda para detectar el nivel de retroalimentación que existe entre el facilitador y los estudiantes. El 9.9\% dijo que muy frecuentemente; para el $26.8 \%$ de los alumnos, frecuentemente; ocasionalmente el $34.5 \%$; rara vez, un $17.6 \%$; el $6.3 \%$ opinó que nunca y el $4.9 \%$ no contestó. Ver figura 8

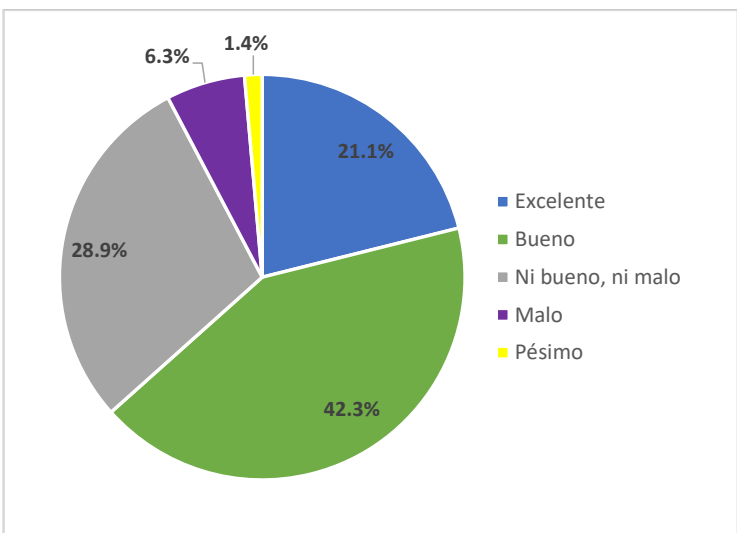

Figura 7: ¿Como evaluarías a los docentes que imparten en modalidad virtual?

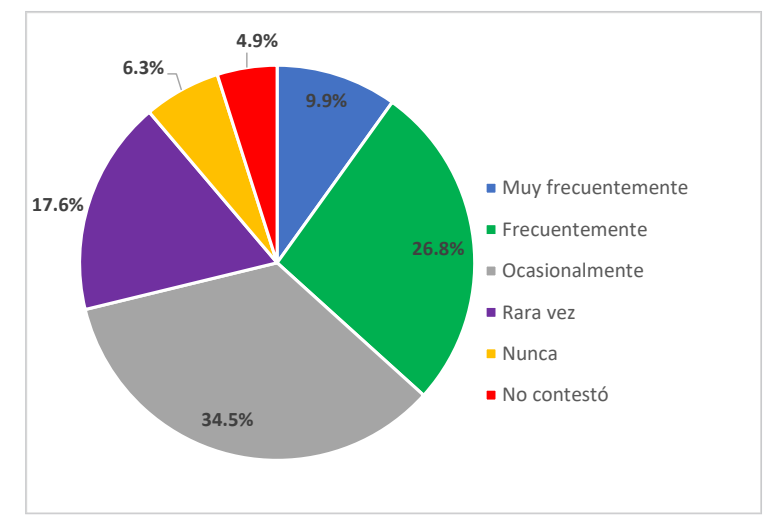

Figura 8: ¿El docente despejó tus dudas durante el curso?

De los doce estudiantes entrevistados de los diferentes programas que imparte la Facultad, siete manifestaron que los docentes sí resolvían sus dudas a tiempo, uno dijo que a veces y cuatro personas dijeron que no. Martín Soto Molina, estudiante del programa educativo Ingeniero en Computación, manifestó: "sí, el docente en línea puedo decir que es más competente que el presencial". José Marino Cabrera dijo: "sí, las veces que le preguntaba siempre me contestaba y me ayudaba mucho". Roberto Rodríguez M. comentó: "no, nunca respondió a mis dudas". Otro estudiante dijo que: "la mayor parte de las dudas no me contestaba"

\section{Desempeño del estudiante}

El estudio independiente trae consigo una serie de responsabilidades y compromisos para el estudiante, a diferencia de la enseñanza presencial donde en la clase hay una interacción directa y una retroalimentación constante con el docente.

En la modalidad virtual recae en un compromiso personal pues el alumno es quien tiene la responsabilidad de entrar a la plataforma y de realizar 
las diferentes actividades que se designan sin una presión más directa del docente.

Se les preguntó a los estudiantes: ¿cómo había sido su experiencia ante un estudio independiente? A lo que el $12.0 \%$ dijo que excelente; $58.5 \%$ dijo que fue bueno; el $21.1 \%$ ni bueno ni malo; el $4.9 \%$ cataloga su experiencia como mala y el $3.5 \%$ dice que fue pésima. Estos últimos, aunque son porcentajes menores, lo catalogan como una experiencia negativa y un porcentaje mayor le es indiferente lo que permite observar cierto grado de inconformidad.

También se les cuestionó sobre su rol y su eficacia como estudiante a través de la pregunta: “¿Cómo fue tu desempeño como estudiante en la unidad de aprendizaje tomada en línea? Ante esta pregunta el $11.3 \%$ dijo que fue excelente; el $56.3 \%$ lo evalúa como bueno; el $24.6 \%$ dice que ni bueno ni malo; el $5.6 \%$ lo cataloga como malo y el $2.1 \%$ piensa que su desempeño fue pésimo. Ver figura 9.

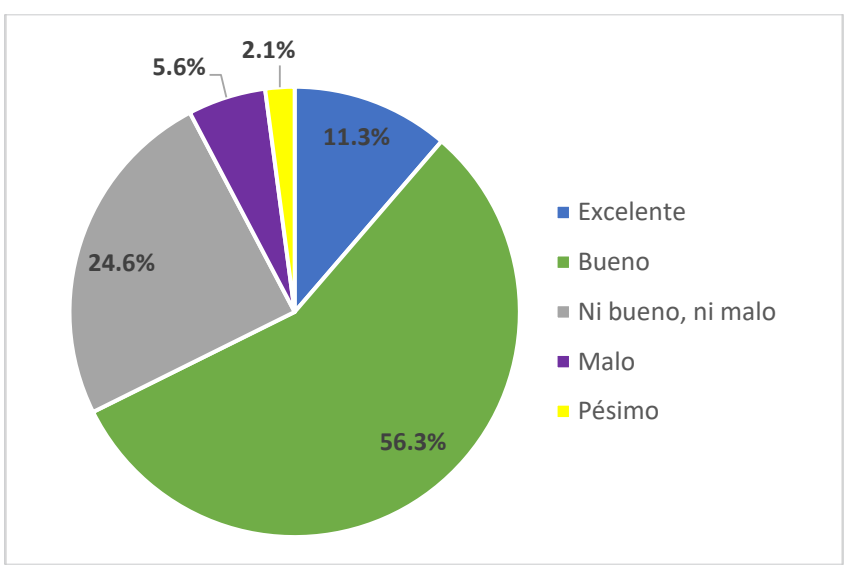

Figura 9: ¿Cómo evalúan los estudiantes su desempeño en el curso virtual?

¿Hubo exceso en la cantidad de trabajos? el $16 \%$ de los estudiantes opinó que muy frecuentemente; $26.1 \%$ dijo que frecuentemente; el $30.3 \%$ opinó que ocasionalmente; el $16.9 \%$ comentó que rara vez; el $5.6 \%$ dijo que nunca, y el $4.2 \%$ no contestó. Ver figura 10

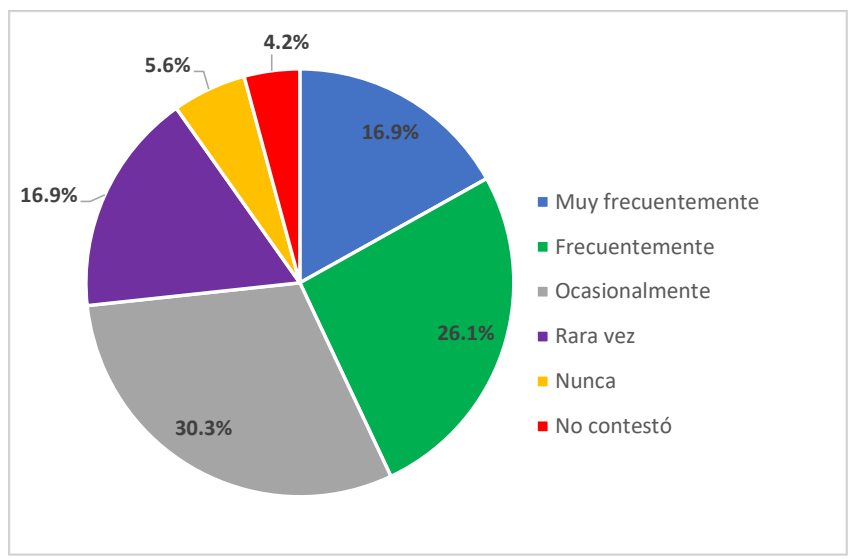

Figura 10: ¿Hubo excesos en la cantidad de trabajos?
En los ítems dinámica del curso y exceso de tareas, la percepción negativa en los estudiantes se incrementa, sobre todo, en este último, hay que considerar que el compromiso autodidacta del alumno y el manejo de la plataforma que algunos la consideraron no tan sencilla, puede influir en su deserción o reprobación.

\section{Evaluación}

La evaluación es uno de los indicadores más importantes en todo programa educativo, ya que es un parámetro para identificar el desempeño y el nivel de las competencias adquiridas por los estudiantes.

Al preguntarles a los alumnos si hubo claridad en los aspectos que se valuarían, el $16.9 \%$ dijo que muy frecuentemente; el $40.8 \%$ dijo que frecuentemente; el $28.2 \%$, ocasionalmente; el $6.3 \%$ dijo que rara vez; el $3.5 \%$ que nunca y un $4.2 \%$ no contestó.

Sobre ¿Qué les pareció el sistema de evaluación? al $15.5 \%$ le pareció excelente; el $50.7 \%$ bueno; el $24.6 \%$, ni bueno ni malo; el $6.3 \%$, malo y el $2.8 \%$, pésimo.

Se les preguntó si tuvieron algún problema en la asignación de su calificación durante los cursos que han tomado en línea, a lo que el $10.6 \%$ dijo que muy frecuentemente; $12.0 \%$ dijo que frecuentemente; el $16.9 \%$ manifestó que ocasionalmente; el $14.1 \%$ dijo que rara vez; el $42.3 \%$ manifestó que nunca y el $4.2 \%$ no contestó.

Aunque los porcentajes negativos no son altos, aun así, demuestran una insatisfacción en un sector del alumnado que está cursando o cursó en modalidad virtual alguna de sus unidades de aprendizaje en estos los últimos ítems.

Al respecto, se detectó que hay un mayor índice de abandono que de reprobación, de acuerdo con información proporcionada por trabajadores de la Coordinación de Educación Virtual. Diez de los doce estudiantes entrevistados manifestaron que volverían a tomar de nuevo una materia en línea y estarían dispuestos a invitar a un amigo a hacerlo, sin embargo, dos dijeron lo contrario. Es necesario considerar las repuestas en la encuesta sobre el exceso de tareas y las dinámicas del curso que son aspectos a tomar en cuenta para solucionar esa inconformidad que aunque mínima pudiera ir en aumento.

\section{Aprendizajes esperados y objetivos cumplidos}

Para esta subcategoría se les preguntó a los estudiantes si creen que se adquieren las mismas competencias tomando la unidad de aprendizaje en línea que de manera presencial, a lo que $12.7 \%$ dijo que muy frecuentemente; frecuentemente, el $30.3 \%$; el $23.9 \%$ dijo que ocasionalmente; el $14.1 \%$ dijo que rara vez; el $13.4 \%$, nunca y no contestó el $5.6 \%$.

Si analizamos solo los porcentajes negativos, acumulan un $51.4 \%$ que es poco más de la mitad de los encuestados, sin contar que el $5.6 \%$ que no contestó 
es un porcentaje que evita el tema y no está seguro de su respuesta. Este porcentaje contrasta un poco con los resultados positivos, al preguntar si creen que se cumplieron los objetivos del curso en línea, el $16.9 \%$ manifestó que muy frecuentemente; el $46.5 \%$ dijo que frecuentemente; el $18.3 \%$ dijo que ocasionalmente; el $9.9 \%$, rara vez; $4.2 \%$ dijo que nunca y el $4.2 \%$ no contestó a la pregunta.

La estudiante Karla Juárez Mata del programa educativo de Ingeniería en Computación piensa que no se adquieren las mismas competencias de manera virtual que presencial: "no porque de manera presencial tu compartes tus ideas y tus conocimientos con el resto de la clase, y de forma virtual es más lo que piensas y lo que viene ahí (plataforma) y el maestro te lo acepta o no".

Martín Soto Molina, estudiante del programa educativo Ingeniería en Computación, comenta que, por el contrario: "en algunos de los casos hasta es mejor tomarla en línea porque siguen el Plan de Estudios y de forma presencial a veces no cumplen con eso, así que puedo decir que sí". El estudiante José Contreras Castro coincide al respecto: "Si le demuestras demasiado interés y estudias ahí en virtual, sí, adquieres casi los mismos conocimientos".

Para el $13.4 \%$ de los alumnos, los aprendizajes adquiridos en la modalidad virtual fueron excelentes; el $66.9 \%$ los cataloga como buenos; el $13.4 \%$ piensa que ni buenos ni malos; el $5.6 \%$ considera que fueron malos y sólo el $7 \%$ dice que fue pésimo.

Se les preguntó a los estudiantes si la unidad de aprendizaje virtual cumplió con sus expectativas. El $62.2 \%$ manifestó que sí; el $17.6 \%$ dijo que no; el 16.2 $\%$ dijo que no está muy seguro ya que dijo optó por la respuesta de no sé y el $4.2 \%$ no contestó.

Los docentes de este sistema educativo en línea manifiestan que es mejor el aprendizaje virtual y que los estudiantes sí logran las competencias al igual que si tomaran una unidad de aprendizaje presencial.

\section{Conclusiones}

Lograr la satisfacción del estudiante en las universidades es uno de los objetivos principales dentro de su crecimiento y desarrollo como una institución generadora de conocimiento, pues es su razón de ser. La satisfacción es el resultado de un proceso valorativo el cual transcurre en un proceso de construcción de significados y sentidos personales (Gento y Vivas, 2003), por consiguiente, si una persona expresa estar satisfecha o no con respecto a algo, está emitiendo un juicio de valor a partir de un proceso de evaluación.

Pérez y Pereyra (2015), exponen que sistematizar un sondeo sobre la satisfacción en el ámbito universitario puede indicar cómo se está generando el proceso de enseñanza-aprendizaje y podría considerarse como una medida de control de calidad de dicho proceso. De ahí la importancia de impulsar este tipo de estudio para detectar indicadores que permitan mejorar los procesos en este ámbito.

Este juicio de valor se pudo identificar en los resultados de las categorías contempladas en la investigación, al medir la satisfacción estudiantil en los cursos de educación virtual como resultado de todo un proceso, así podemos afirmar que el trabajo de la Coordinación General de Educación Virtual de la UAGro es positivo y bien aceptado por los estudiantes, no obstante, se detecta que uno de los indicadores más importantes para elevar los índices de satisfacción en esta modalidad de enseñanza-aprendizaje es el proceso de comunicación que se da entre estudiantes y docentes, que incluye los canales utilizados y el tipo de retroalimentación.

Estamos convencidos de que la educación no puede aislarse de la revolución en la comunicación y la tecnología que está permeando todos los ámbitos, y exige, sin lugar a dudas, modificar los procesos de comunicación en el contexto educativo, así como las metodologías pedagógicas tradicionales para adaptarlas a las necesidades de los estudiantes del nuevo milenio. (Manrique, 2015). Las opiniones de los estudiantes arrojan que los docentes no se distinguen como entes impulsores que estimulen un aprendizaje más eficaz. Más de la mitad de los estudiantes encuestados no perciben a sus facilitadores como motivadores del proceso de enseñanza aprendizaje, aunado a la poca retroalimentación que ellos ofrecen, lo que afecta el estímulo para aprender y el clima de confianza que pudiera generarse.

Los cambios tecnológicos conllevan nuevos canales de comunicación los cuales el docente debe conocer y apropiarse, asimismo, debe valorar el proceso comunicativo como un factor esencial que estimula y contribuye con el logro de la culminación satisfactoria del aprendizaje en línea. García Aretio (1999), enfatiza que la interacción en la educación a distancia puede adoptar diversas modalidades en función de la intermediación, del tiempo y del canal. Sin embargo, no se observa un buen uso de estas modalidades de acuerdo a la percepción de los estudiantes, detectada en las entrevistas semi estructuradas y en la encuesta. El contacto poco frecuente entre alumno y docente provoca una insatisfacción en un porcentaje elevado de estudiantes debido a que lo perciben como escaso, nulo o de baja calidad. Sin embargo, se detecta que son los monitores académicos los que cumplen una función determinante, ya que impulsan a los estudiantes durante todo el proceso formativo, lo que contribuye de manera positiva en que culminen su unidad de aprendizaje de forma satisfactoria, puesto que resuelven dudas y los motivan constantemente, lo que influye en reducir el nivel de deserción y reprobación. En este mismo sentido, se manifestado que, a pesar de que la unidad de aprendizaje es en línea, no es el correo electrónico, ni la plataforma el medio más utilizado para el contacto con los facilitadores o el monitor académico, sino el WhatsApp.

Una de las barreras de comunicación que se detecta es la misma tecnología que, al no saber utilizarla, en el caso de los estudiantes, se convierte en un obstáculo 
que limita y dificulta la comunicación. Al respecto, retomamos las sugerencias de Hernández (2008) quien considera que el docente de cursos virtuales, como asesor constructivista en línea, ha de desarrollar múltiples actividades, como: reflexionar acerca de su grado de compromiso no solo con sus estudiantes, sino consigo mismo; saber programar acciones interactivas; reconocer que no todos aprenden de la misma forma ni al mismo ritmo; propiciar la participación social y los hábitos de estudio entre los alumnos, y salvar progresivamente las dificultades con el uso de la computadora. La mayoría de los estudiantes objeto de estudio no perciben estos aspectos en sus docentes en línea.

Con respecto a la autoevaluación del alumno sobre su desempeño en el curso: la dinámica de trabajo, el exceso de tareas, en el caso de algunas unidades de aprendizaje, y el manejo de la plataforma que para algunos fue considerada como no tan sencilla, son aspectos que pueden influir en el abandono del curso que, de acuerdo con información proporcionada en la Coordinación de Educación Virtual, es superior al porcentaje de reprobación. Se puede afirmar, entonces, que aspectos como el acceso a la tecnología, el dominio que pueden tener de ella los alumnos, el diseño de la plataforma educativa, la calidad de su contenido y su practicidad impactan en la percepción y satisfacción de los estudiantes sobre el curso. Aunque un porcentaje de estudiantes fue indiferente al contestar y su respuesta no fue positiva, es primordial atender a los que tienen un punto de vista negativo ante esta experiencia educativa, por mínimo que sea este sector, ya que de lo contrario se puede incrementar esta percepción.

Es importante recalcar que el estudio independiente viene a cambiar los paradigmas clásicos de la educación tradicional y trae consigo una serie de responsabilidades para el estudiante y su compromiso como autodidacta, de la misma forma, el docente debe ser un facilitador que incorpore a su trabajo diario los aportes de teorías del aprendizaje aplicadas a la tecnología como el constructivismo, que permite impulsar los aprendizajes significativos, colaborativos, y la relación con los aprendizajes previos del alumno. Por todo esto, es imprescindible que los docentes del siglo XXI asuman este gran reto frente a un proceso de enseñanza aprendizaje que va cambiando debido a los avances tecnológicos, lo que exige una actualización pedagógica constante para ser facilitadores que promuevan el desarrollo y las expectativas del estudiante del nuevo milenio.

\section{Referencias}

Acuña, A. (2012). El uso de las tecnologías de la información y la comunicación (TIC) en la educación. México: Cuadernos de la casa ANUIES
Alonso, L. y Blázquez F. (2012). El docente de educación virtual. Guía Básica España: Narcea

Barraza, A. (2002). Constructivismo social: un paradigma en formación, En PsicologíaCientifica. com Artículo Publicado el 02 de abril de 2002, pp. 1-14 Recuperado de: http://ww2.educarchile.cl/ UserFiles/P0001/File/constructivismo-social-unparadigma-en-formacion.pdf

Gallardo, E. (2012). Hablemos de estudiantes digitales y no de nativos digitales en Revista de Ciències de l'Educació Juny 2012. Pag. 7-21 Recuperado de:https://revistes.urv.cat/index.php/ute/article/ viewFile/595/574

García, A. (1999). Fundamentos y Componentes de la Educación a Distancia. Revista Iberoamericana de Comunicación a Distancia Vol 2 N.2 mp. 55 Recuperado de:http://www.biblioteca.org.ar/ libros/142121.pdf

Gento S. y Vivas M. (2003). El SEUE: un instrumento para conocer la satisfacción de los estudiantes universitarios con su educación en: Acción pedagógica, Vol. 12, No. 2 / 2003 pp.16-27 Recuperado de file:///C:/Users/Toshiba/Downloads/ Dialnet-EISEUE-2972060.pdf

De la Fuente, H., Marzo, M. y Reyes, M. (2010). Análisis de la satisfacción universitaria en la Facultad de Ingeniería de la Universidad de Talca en Ingeniare. Revista Chilena de Ingeniería, vol. 18 № 3, 2010, pp. 350-363 Recuperado de: https:// scielo.conicyt.cl/scielo.php?script=sci_arttext\&pid $=$ S0718-33052010000300009

Díaz, S. (2009). Plataformas educativas, un entorno para profesores y alumnos en Temas para la educación. Revista profesional para profesionales de la enseñanza. N.2 mayo 2019 pp.1-5 Recuperado de: https://www.feandalucia.ccoo.es/docu/p5sd4921. pdf

Díaz, F. y Hernández, G. (2010). Estrategias docentes para un aprendizaje significativo. Una interpretación constructivista. Tercera edición. México: Mc Graw Hill.

Hernández, S. C. (2007). El constructivismo social como apoyo en el aprendizaje en línea en Nueva época. Año 7 / Núm. 7 Noviembre 2007 pp. 46-62 Recuperado de: http://www.redalyc.org/articulo. oa? $\mathrm{id}=68800705$

Hernández, S. (2008). El modelo constructivista con las nuevas tecnologías: aplicado en el proceso de 
aprendizaje en Revista de Universidad y Sociedad del conocimiento vol. 5 n. ${ }^{\circ} 2$ (2008) pp. 1-10 Monográfico [Comunicación y construcción del conocimiento en el nuevo espacio tecnológico] Recuperado de: http://www.uh.cu/static/documents/ STA/EI\%20modelo\%20constructivista\%20TIC.pdf

Hernández Sampieri, R., Fernández, C., y Baptista, P. (2014). Metodología de la investigación MCGrawHillEducatión, México Recuperado de: https://www.uca.ac.cr/wp-content/uploads/2017/10/ Investigacion.pdf

Linares, M y Santovenia, J. (2012). Buenas prácticas, Comunicar e informar. Cuba: Editorial Academia.

Manrique, K. (2015). La web 2.0 y sus servicios como herramientas en el entorno educativo del siglo XXI en Revista digital Universitaria 1 de septiembre de 2015 Vol. 16 Núm. 9 pp.1-13 Recuperado de: http:// www.revista.unam.mx/vol.16/num9/art76/art76.pdf

Mejías, A. y Martínez, D. (2009). Desarrollo de un Instrumento para Medir la Satisfacción Estudiantil en Educación Superior en Docencia Universitaria, Volumen X, No 2, Año 2009 http://www.ucv.ve/ fileadmin/user_upload/sadpro/Documentos/ docencia_vol10_n2_2009/5_mejias_agustin_y_ martinez_domelis.pdf

Moodle (2018). Filosofía. Recuperado de: https://docs. moodle.org/all/es/Filosof\%C3\%ADa

Pérez, I. y Pereyra, E. (2015). Satisfacción estudiantil: Un indicador de la calidad educativa en el Departamento de Biología Celular, UCV en Revista de Pedagogía, vol. 36, No99, 2015, pp. 69-89 Recuperado de http:// www.redalyc.org/html/659/65945575008/

Piscitelli A. (2009). Aula XXI Nativos digitales. Dieta cognitiva, inteligencia colectiva, y arquitecturas de la participación. Argentina: Santillana. Recuperado de:https://issuu.com/santillanaargentina/docs/ nativosdigitales

Prensky, M. (2001). Nativos Digitales, Inmigrantes Digitales, en Aprender a pensar, 18 de mayo del 2009, tomado de On the Horizon (MCB University Press, Vol. 9 No. 6, December 2001) Recuperado de: https://aprenderapensar.net/2009/05/18/nativosdigitales-vs-inmigrantes-digitales/

Rodríguez, A. y Pérez, A. (2018). Satisfacción del egresado respecto de su formación profesional en Cuaderno de Pedagogía Universitaria Vol. 15, no.29, enero-junio 2018 pp.12-30 República
Dominicana. Recuperado e: http://cuaderno. pucmm.edu.do/index.php/cuadernodepedagogia/ article/view/297/274

Ruiz, A. (2008). La muestra: algunos elementos para su confección. Recuperado de: http://www.raco.cat/ index.php/reire/article/viewFile/121055/166930

Salinas, A., Morales, J. y Martínez P. (2008). Satisfacción del estudiante y calidad universitaria: un análisis explicatorio en la unidad académica multidisciplinaria agronomía y ciencias de la universidad autónoma de Tamaulipas, México. Revista de Enseñanza Universitaria 2008, N. ${ }^{\circ} 31$; 39-55 Recuperado de: http://institucional.us.es/ revistas/universitaria/31/4SalinasGuti.pdf

Tünnermann, C.(2011). El constructivismo y el aprendizaje de los estudiantes UDUAL, en Universidades México, n. 48, enero - marzo 2011, pp. 21 - 32 Recuperado de: http://www.redalyc.org/ pdf/373/37319199005.pdf 